\title{
Identification of pigments in artworks by inverse tangent derivative of spectrum and a new filtering method
}

\author{
F. Fazlali and S. Gorji Kandi ${ }^{*}$ (i)
}

\begin{abstract}
Employing an economical and non-destructive method for identifying pigments utilized in artworks is a significant aspect for preserving their antiquity value. One of the non-destructive methods for this purpose is spectrophotometry, which is based on the selected absorption of light. Mathematical descriptive methods such as derivatives of the reflectance spectrum, the Kubelka-Munk function and logarithm have been employed for the characterization of the peak features corresponding to the spectrophotometric data. In the present study, the mentioned mathematical descriptive methods were investigated with the aim to characterize the constituents of an Iranian artwork but were not efficient for the samples. Therefore, inverse tangent derivative equation was developed on spectral data for the first time, providing considerable details in the profile of reflectance curves. In the next part, to have a simpler and more practical method it was suggested to use filters made up of pure pigments. By using these filters and placing them on the samples, imaging was done. Then, images of samples with and without filter were evaluated and pure pigments were distinguished. The mentioned methods were also used to identify pigments in a modern Iranian painting specimen. The results confirmed these methods with reliable answers indicating that physical methods (alongside chemical methods) can also be effective in determining the types of pigments.
\end{abstract}

Keywords: Spectrophotometry, Mathematical description, Inverse tangent derivative, Imaging, Pigment identification, Artwork

\section{Introduction}

There are several complementary analytical techniques to provide an understanding of the composition of the materials in artworks that can be classified into destructive and non-destructive. Non-destructive techniques such as spectrophotometry, Fiber Optic Reflectance Spectroscopy (FORS) [1-4] and Raman [5-9] provide an easily accessible and safe means to observe various components of a painting. Spectrophotometry presents spectral information in various archaeological, artistic, medical, biochem ical sciences [10], and artworks [1, 11], revealing distinctive features not visible to the naked

*Correspondence: s.gorji@aut.ac.ir

Polymer Engineering and Color Technology Department, Amirkabir University of Technology, Tehran, Iran eye. The reflection spectrum of each pigment is specific for that individual pigment, the same as fingerprint for human [12].

Spectrophotometry operates based on selective light absorption and diffusion. While absorption of light is a relatively straightforward phenomenon, diffusion is more complex since it depends on the pigment specifications such as particle size, angle of impact, suspending medium, and difference in refractive index of pigment [12-14]. Some spectrophotometers such as FORS methodology provides the possibility to collect reflectance spectra from very small areas of the surface of paintings. Furthermore, this technique is easily portable, lightweight, compact, low-cost, high speed, and easy to use $[15,16]$. This identification method is very useful for the characterization of pure pigment in artworks [11]. 
It is essential to compare the spectra of investigated unknown materials with those available in the database. It should also be noted that pigments and binders may be affected by chemical reactions over time, leading to alterations in their color, for instance, becoming yellowish [17, 18]. Thus, choosing an appropriate database is inevitable [19].

The concentration of pigments can be estimated to some extent by converting the reflectance spectrum to the absorption spectrum using the Kubelka-Munk theory. This is achievable due to the linear relationship between concentration and absorption. In some cases, the accurate identification of the pigment becomes difficult due to the similarity of the reflection spectra of database and the sample. In order to increase the peak intensity and clarify its details, the derivation and logarithm of the reflection spectrum and the Kubelka-Munk theory are taken into account [20-22]. Many researchers studied in Kubelka-Munk space, including Berns, who worked on the mixture of linear combinations of pure pigments [23] and then Dupuis and Menu, who investigated mixing pigments with different concentrations in organic binders [14]. Rodriguez and Fernández provided another method for classifying pigments in the mixture using the second derivative of Kubelka-Munk [20]. The most common used methods are checking the height of first and second derivatives of peaks, which illustrates the characteristics of the reflectance spectrum, reveals the details better, and eliminates unnecessary information such as static linear shifts [20]. Pallipurath et al. developed a method for determining the characteristics of the first derivative of the spectrum for different pigments by analyzing the peak feature corresponding to color transition edges. They showed how the ratio of the areas under the characteristic peaks ascribed to different pigments could be used to quantify the composition of binary paint mixture [24]. In another study, Szalai et al. identified iron minerals using the first and second derivatives of the Kubelka-Munk theory [22]. In general, for the identification of pigments, four analyses have been used: (1) color descriptions with the aid of color space such as CIELAB and Munsell, (2) Kubelka-Munk analysis, (3) Derivative methods [20], (4) Hyperspectral and multispectral imaging methods. The latter mentioned method is proposed based on the spectral and spatial picture of a detected object [25-28]. A spectral imaging approach includes the application of filters in front of camera [29-31]. Cosentino investigated identification of pigments in different hues by using a filter set in different light condition [32].

\section{Research aim}

The aim of our research was to employ mathematical algorithms and evaluation of a new equation on spectroscopy data for rapid, uncomplicated, and nondestructive detection of artwork pigments. In addition, new glassy filters of pure pigment were characterized to be placed on the sample, and pigment identification was carried out by capturing images of samples. The results obtained from these methods have simpler analysis compared to hyperspectral and multispectral imaging.

\section{Materials and methods}

\section{Sample preparation}

In this study, twenty-one commercial inorganic pigments were purchased from the pigment markets, which seemed to be the most widely pigments in the Iranian artworks. The utilized pigments in this research varied in their chemical structure and included iron oxide, molybdate, and chromium oxide. Linseed oil was used as the binder and cobalt dryer was employed to accelerate the drying process of the samples. Every pure pigment was blended with linseed oil and drying agent. A film applicator was used to apply films with 60 microns thickness on the papers. Therefore, twenty-one films of pure pigments were prepared. Then, mixtures of pigments with ratios of 1:1 and 2:1 were made of seven primary hues (red, yellow, blue, green, ochre, orange and white), and were applied on papers as mentioned. Finally, fifty-seven samples with a size of $2 \mathrm{~cm} \times 2 \mathrm{~cm}$ were prepared and placed on a color palette. Figure 1a shows the color and the codes of pure and mixed pigments samples.

\section{Filter preparation}

Some paints made in the previous step were combined with a large amount of linseed oil to create transparent specimens. Then they were applied as 30 microns thick films on glass substrates, which were degreased with soap and acetone prior to applying the films. The applied films

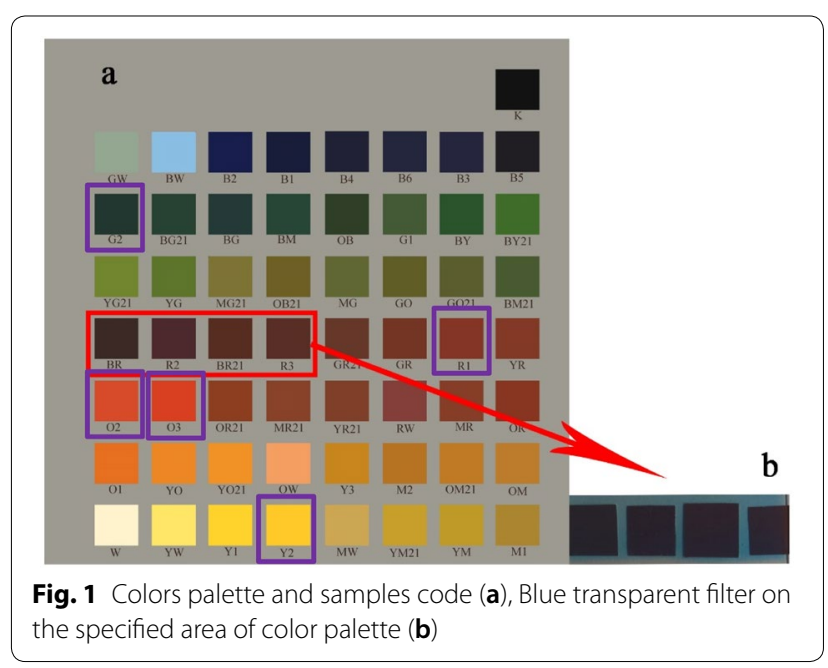


were kept in the ambient condition of lab for a month to complete their drying process. After that, they were individually placed on a color palette, and then a photo was taken. Figure $1 \mathrm{~b}$ shows the blue transparent filter on the color palette.

\section{Spectrophotometry and data processing}

A GretagMacbeth Color-EYE 7000A spectrophotometer was used in SCE mode within the visible range from $360 \mathrm{~nm}$ to $750 \mathrm{~nm}$ in $10 \mathrm{~nm}$ steps for measuring reflectance spectra of pure and mixed pigments.

Some mathematical operations need to be applied on spectral data to enhance the sensitivity of the curves. In the current study, the first and second derivatives of the reflectance spectrum [6], the first and second derivatives of the Kubelka-Munk function [20, 21, 23], and the logarithm of the Kubelka-Munk function [19] were employed. However, the result curves showed that these operations were not applicable to our samples. Therefore, the inverse tangent derivative equation was applied on spectral data in this study. According to Eq. 1, for a considerable change in the horizontal axis $(x)$, the vertical axis (y) changes slightly and demonstrates details very clearly (Fig. 2).

$$
\frac{d}{d x} \arctan (x)=\frac{1}{1+x^{2}} .
$$

By using this equation, the intensity changes of spectral reflectance were more obvious in short wavelengths. Considering the slight yellowish color of the binder used in this study, the spectrum curves are affected in short wavelengths [11] and the proposed equation confirms that. As can be seen in Eq. 2, in which $R$ and $\lambda$ are

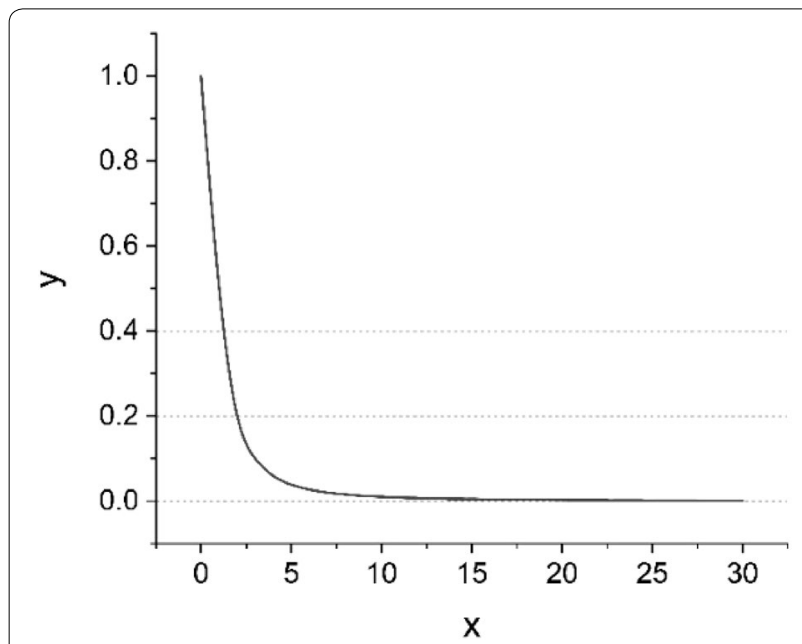

Fig. 2 Inverse tangent derivative graph reflectance and wavelength, respectively, the $\mathrm{F}(R)$ has an inverse relation with wavelength, so the quantity of $\mathrm{F}(R)$ is greater in short wavelengths. This new scale is useful to depict a wide range of values more distinguishable than a linear scale.

$$
F(R)=R /\left(1+\lambda^{2}\right)
$$

In the second and higher derivatives, the variations of reflection at short wavelengths are not specified, whereas, Eq. 2 determines the variations more noticeably.

In the imaging part, a Nikon D5300 digital camera was used to capture the images of the prepared samples. The camera was placed at a specified distance of $50 \mathrm{~cm}$ from the samples in a light cabinet with medium gray walls and was calibrated. So that all shooting conditions were adjusted to be constant. The images were captured under D65 light source.

In the filtering method, highly transparent and nonreflective filters from the same pigments were produced. They were individually placed on the color palette, and then the image of every sample was captured.

Microsoft Excel and MATLAB 2016 were used as software tools to apply mathematical description on reflectance data. In addition, findpeak function was applied on inverse tangent derivative to illustrate the wavelengths with maximum peak computationally.

\section{Goodness fitting coefficient}

For comparison of curves, Goodness of Fit Coefficient (GFC) [33] has been reported. GFC metric is calculated by the following equation (Eq. 3), where $R$ and $R^{\wedge}$ are the pigments spectral reflectance of sample and reference, respectively. The values of the GFC range from zero to one, where one indicates a perfect matching. On the other word, the farther it goes from one, the less similar it becomes.

$$
G F C=\frac{\left|\sum_{\lambda} R_{\lambda} R_{\lambda}^{\wedge}\right|}{\sqrt{\left|\sum_{\lambda} R_{\lambda}^{2}\right|} \sqrt{\left|\sum_{\lambda} R_{\lambda}^{\wedge 2}\right|}}
$$

\section{Calibration}

For the capturing and processing of the images, the camera was calibrated by a white paper that was placed near the color palette for each shot. The paper adjusts white balance, exposure, and saturation after the image has been captured. Furthermore, all images were captured under a D65 light source with fixed manual conditions and no flash. 


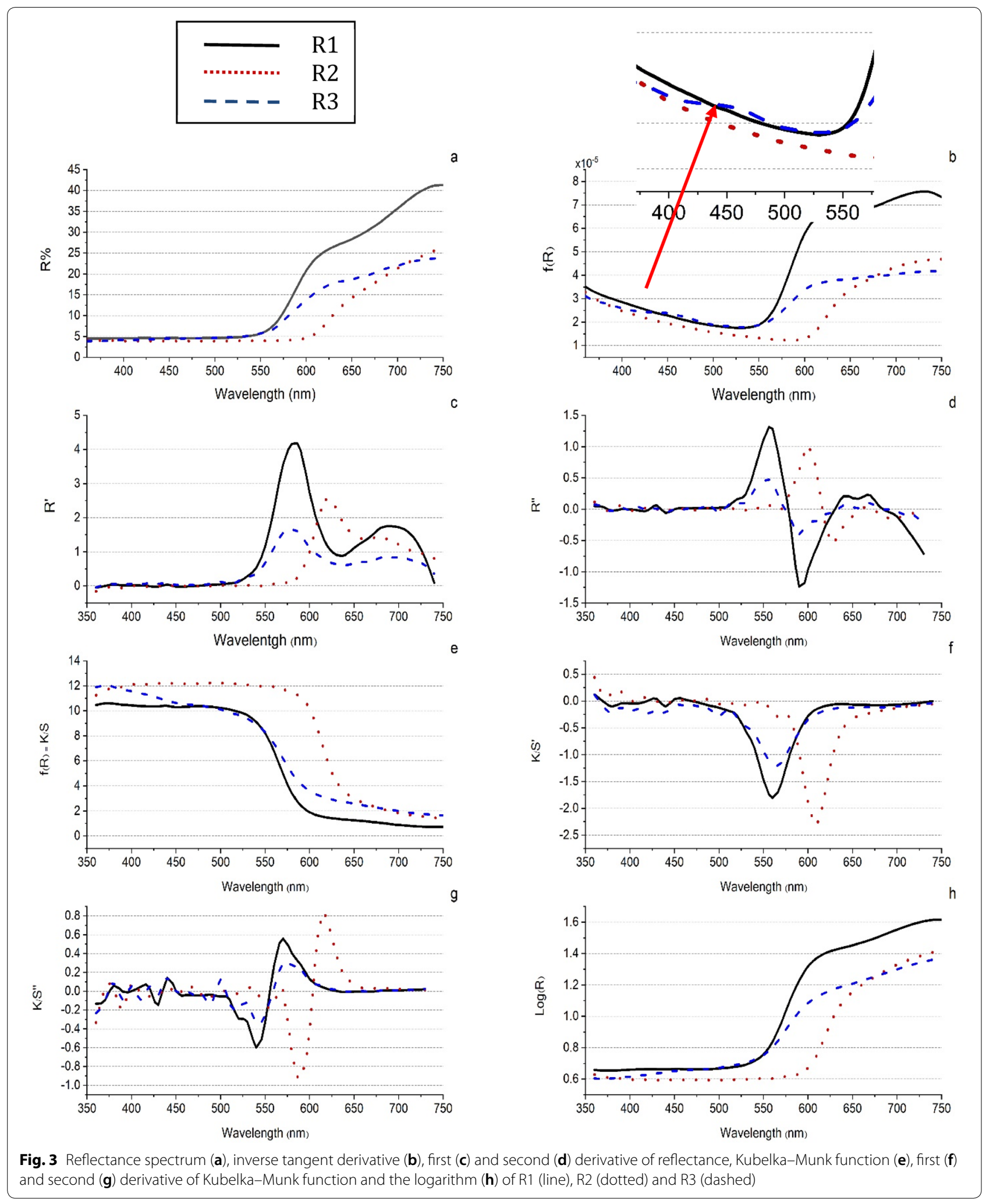




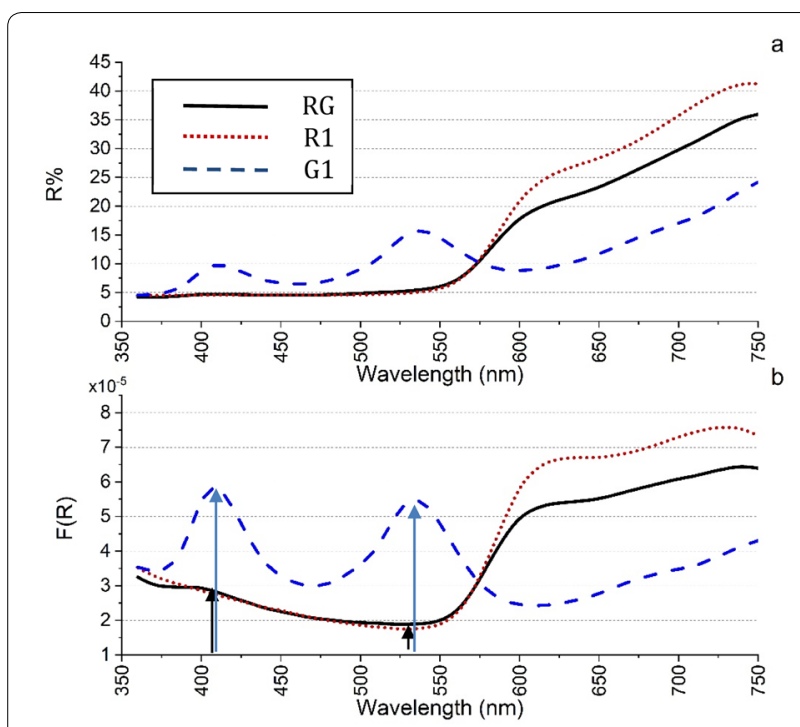

Fig. 4 Reflectance spectrum (a) and inverse tangent derivative (b) of RG (line), R1 (dotted) and G1 (dashed)

\section{Results and discussion}

The results are described in two parts, including spectral analysis and filtering method.

\section{Identification of pigments by spectrophotometry}

In the first part, the identification of pigments was done using their spectral data. The samples were separated based on hue, and then mathematical descriptions were

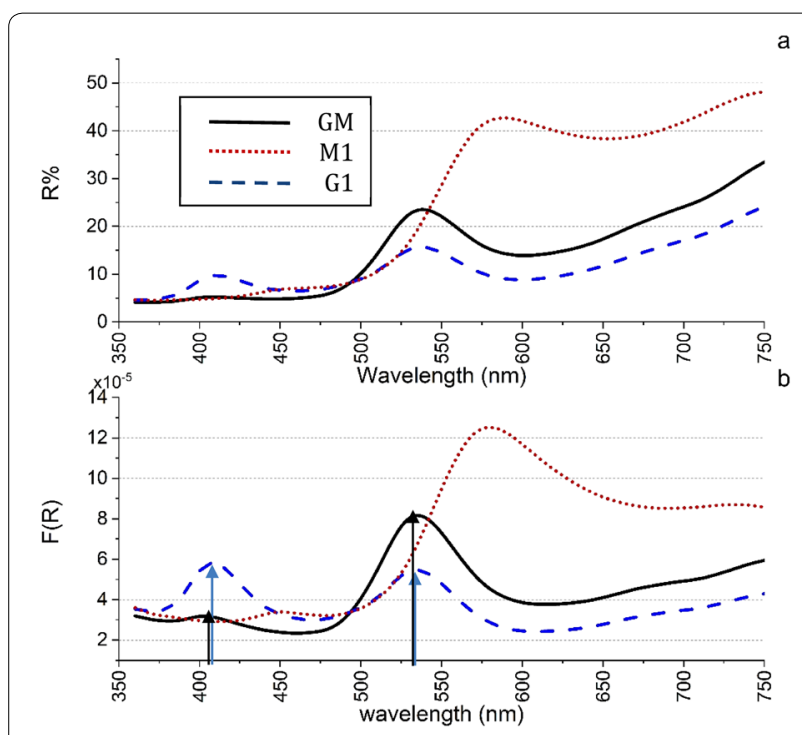

Fig. 5 Reflectance spectrum (a) and inverse tangent derivative (b) of GM (line), G1 (dashed) and M1 (dotted) pigments

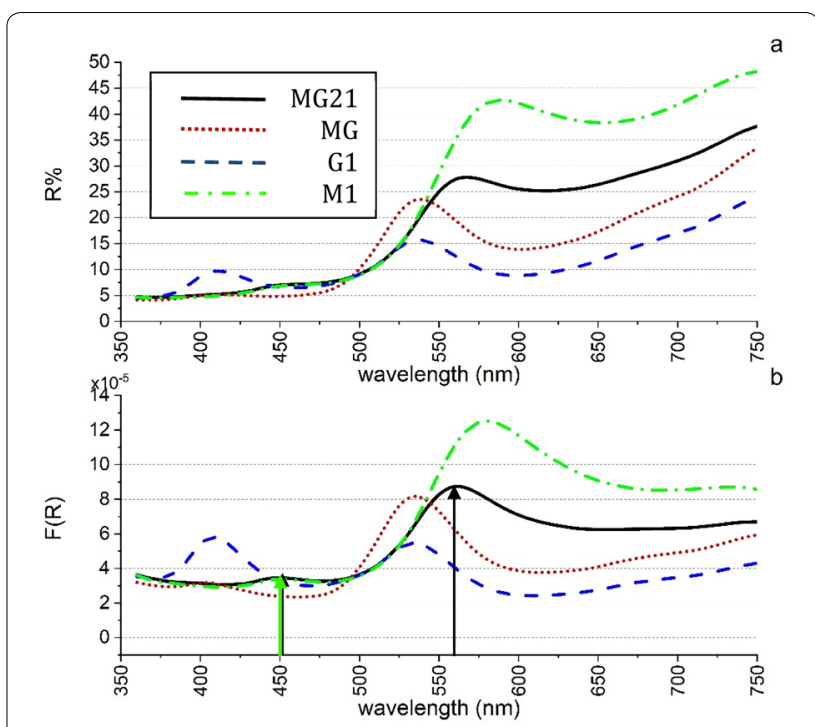

Fig. 6 Reflectance spectrum $\mathbf{a}$ and inverse tangent derivative $\mathbf{b}$ of MG21 (line), MG (dotted), G1 (dashed), M1 (dash-dotted) pigments

applied to their reflectance spectrum in which the details of each pigment would be distinguishable and distinct.

In the case of pigments having the same hue, it is difficult to distinguish their reflectance curves' difference. For instance, according to Fig. 3a, the employed red pigments in this study had the same reflecting curves of $\mathrm{R} 1, \mathrm{R} 2$, and R3 in the range of $580 \mathrm{~nm}$ to $750 \mathrm{~nm}$ with various intensities. Thus, it is not easy to distinguish the differences between them. For the sake of clarifying the details, mathematical descriptions were used. As shown in Fig. 3c, R1 and R2 have different maximum peaks and $\mathrm{R} 1$ and R3 have same peaks in $580 \mathrm{~nm}$ and $690 \mathrm{~nm}$, while $\mathrm{R} 1$ is molybdate and R3 is ochre. Their detailed difference appears in the inverse tangent derivative (Fig. 3b), in which R3 has a small peak in $450 \mathrm{~nm}$. For the other descriptions, the curves are not easy distinguishable as seen in Fig. 3 (c, d, e, f, g, h).

Finally, we investigated the feasibility of pigment identification for several mixtures of pigments. First, all pigments with different hues were mixed in 1:1 (wt \%), and the outcomes were then compared with pure pigments. Due to the different color strength of the pigments, the results were different for each mixture. The higher the color intensity, the stronger the color strength of the pigment. Color strength is the facility with which a colored pigment maintains its characteristic color when mixed with another pigment. Color strength can be determined by absorption coefficient. The absorption coefficient is obtained using Kubelka-Munk function through measuring reflectance spectrum. 


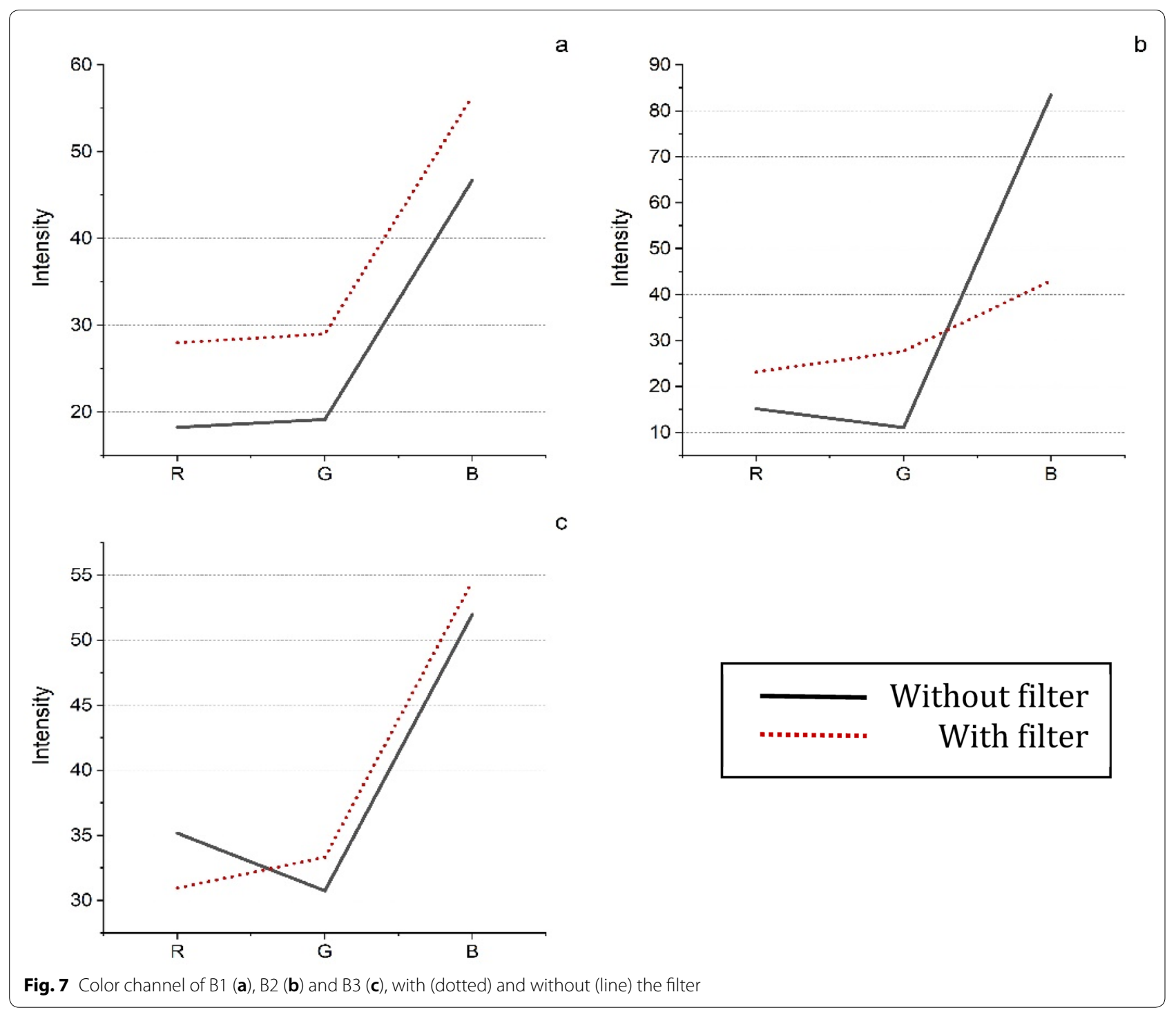

Figure 4a shows the curves of the 1:1 R1 and G1 mixture. As can be seen, their mixture has spectrum similar to the red pigment, and no peaks are corresponding to the green pigment, which indicates a very high color strength of R1. However, the mixture of RG (Fig. 4b) in which inverse tangent derivative is applied on, shows two short peaks in $410 \mathrm{~nm}$ and $530 \mathrm{~nm}$ (based on findpeak function). The achieved result approves the presence of green pigment in the sample.

In another mixture of $\mathrm{G} 1$ and M1, as shown in Fig. 5 (a, b), the reflectance spectrum of the $530 \mathrm{~nm}$ peak indicates the presence of G1, and the reduction in intensity of peak in $410 \mathrm{~nm}$ indicates the presence of M1, which does not have any peak in $410 \mathrm{~nm}$ based on findpeak function. In this mixture, the color strength of both pigments is almost the same as another curve.
In the next part, the proportion of pigments was changed, and mixture of pigments with 2:1 ratio was evaluated. As shown in Fig. 6 by changing the ratio of pigment's concentration, the MG21 (line) curve inclines to $\mathrm{M} 1$ curve. It confirms that the effect of G1 decreases. In fact, the maximum peaks of MG21 in $450 \mathrm{~nm}$ and $560 \mathrm{~nm}$, illustrate that this new mixture peaks tends to M1.

As a matter of fact, adding pigment with less color strength compensates its low intensity and leads the mixture spectrum toward the added pigment curve. The R1 $\left(\frac{K}{S} \lambda_{\max }=10.12\right)$ pigment has much more color strength than all other pigments such as G1 $\left(\frac{K}{S} \lambda_{\max }=6.72\right)$, and M1 $\left(\frac{K}{S} \lambda_{\max }=7.04\right)$. Therefore, even when the ratio is $2: 1$, the spectrum curves change slightly. 


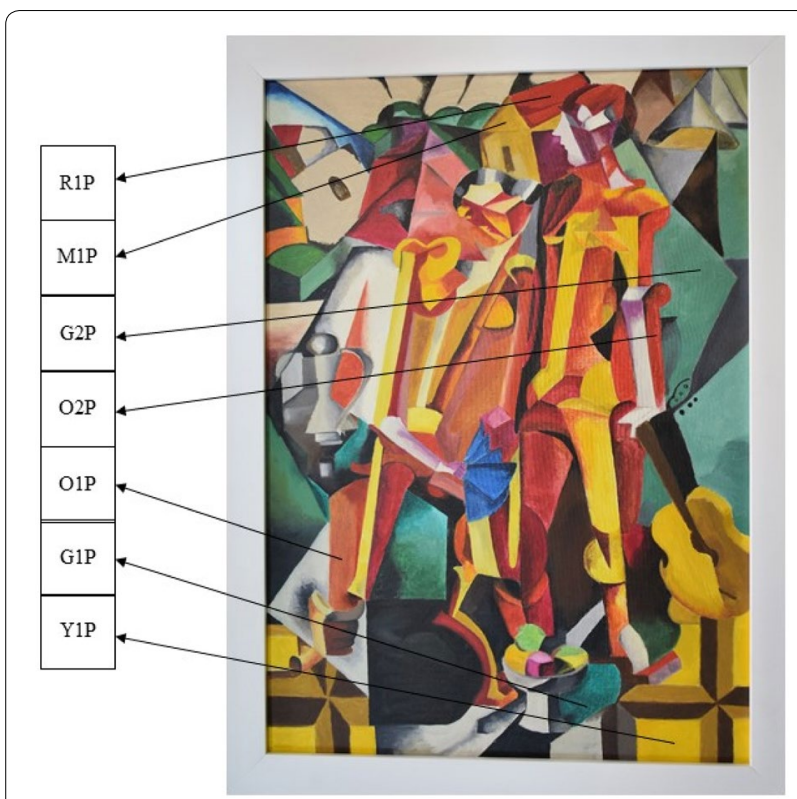

Fig. 8 Painting sample

In these cases, the first and second derivatives of the reflectance spectrum and the Kubelka-Munk function and even the logarithms of each pigment were investigated, and it was inferred that the results sometimes are not recognizable and have obscurity for the investigated samples in this study. However, inverse tangent derivative (Eq. 1), highlights the differences and details of the curves' profiles efficiently (Fig. 3).

\section{Identification of pigments by filtering}

In this step, several filters from the original pure pigments were prepared. Then they were individually placed on the color palette (Fig. 1b). A photo was captured by the camera while a filter was placed on a sample with the same hues. The color channel of the resulting image in different modes was calculated. In Fig. 7, the B1, B2, and $\mathrm{B} 3$ curves are blue pigments without any filter, and the B1F, B2F, and B3F curves are blue pigments with B1 filter. As shown in Fig. 7, the slope of B1 and B1F curves are identical, implying that their color channels change with the same ratio. However, in Fig. 7b, c, the slopes of curves are different, since the filter used for them is not the same as their pigment type. For other pigments, the results were identical.

\section{Application}

A modern Iranian painting was selected in order to evaluate the efficiency of the identification techniques. For this purpose, several color points were investigated. The selected points, namely R1P, M1P, Y1P, G1P, G2P, O1P, and $\mathrm{O} 2 \mathrm{P}$, are illustrated in Fig. 8.

The inverse tangent derivative of the selected points and references were normalized for each hue. As shown in Fig. 9a, and according to findpeak function R1P corresponded to $\mathrm{R} 1$ reference pigment with the maximum peak at $630 \mathrm{~nm}$. As a result, it can be inferred that R1P pigment were identified as red ochre (R1). points were attributed to G2 $(450 \mathrm{~nm}$ and $530 \mathrm{~nm}), \mathrm{Y} 2(570 \mathrm{~nm})$, O2 $(630 \mathrm{~nm})$, and O3 (620) reference pigments (marked with a purple square in Fig. 1a), respectively, as can be seen in Fig. 9b, c, d, e. Since maximum peak detection is not enough to identify the reference pigment, a method that shows the changing procedure in the whole wavelength should be used. Therefore, we employed the GFC method. As a result, at first pigments in the painting were identified by evaluation of maximum peaks and then by GFC value.

The GFC of the points are given in Table 1. As illustrated in Table 1, for Red point, the GFC of R1P:R1 is closer to one in comparison to R2 and R3, which approves the attribution of R1P to R1. In the same way, Y1P, G2P, O1P, and O2P are identified as Y2, G2, O2, and $\mathrm{O} 3$ reference pigments, respectively.

Two M1P and G1P points were not identified with reference pigments in this study, so the database of Cosentino's work was used [34]. G1P was distinguished as Phthalogreen and M1P as Massicot.In order to evaluation of filtering method, the prepared filters were placed on the points with the same hue in the painting and images were captured. Next, the results were compared to the images of the same points without the filter. By using this method, the R1P, Y1P, G2P, O1P, and O2P points were identified since their pigments were the same as the filter's pigment type. As shown in Fig. 10, the slope of R1P and R1F curves are identical, implying that their color channels change with the same ratio. For other points, the results were similar.

\section{Conclusion}

In this research, attempts were made to detect the inorganic pigments used in Iranian paintings by applying non-destructive methods, including spectrophotometry and filtering via providing color films of them. Since spectral curves are usually smooth and difficult to distinguish from each other for similar hues, it is crucial to use mathematical descriptions in the spectral section. Therefore, by using algorithms such as KubelkaMank function, derivatives of the spectrum, and the logarithm, differences in the profile of the spectra were observed. In fact, the details and peaks of each pigment were not very clear and precise in all pigments. 

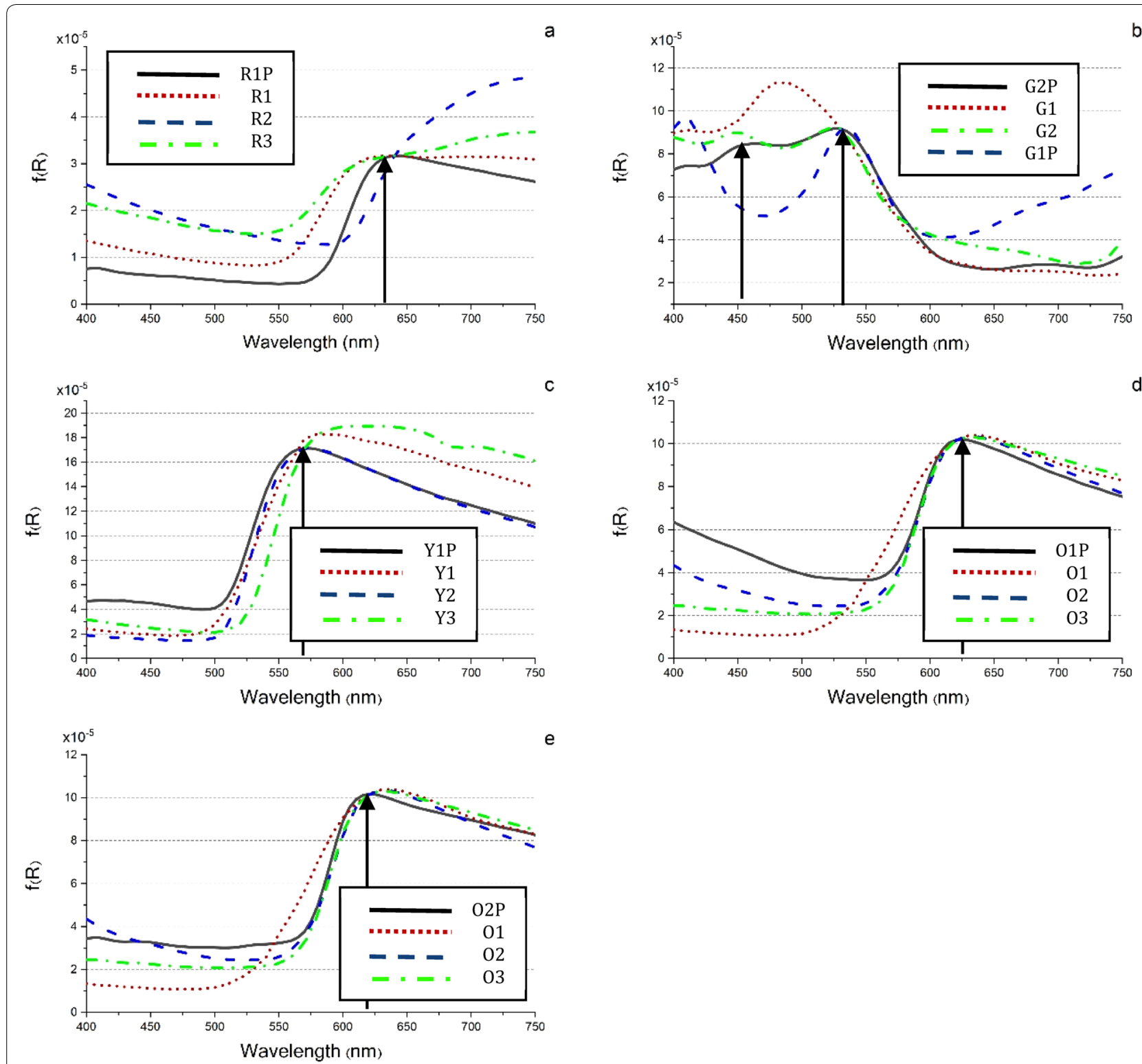

Fig. 9 The inverse tangent derivative of R1P (a), G2P (b), Y1P (c), O1P (d), O2P (e)

However, the newly introduced equation (i.e., inverse tangent derivative) was efficient in illustrating the differences and details of the curves. This method is very accurate and reliable for pure pigment samples. Using it for a mixture of pigments showed that there is a direct relationship between concentration and strength of the

Table 1 Goodness fitting coefficient of points in painting and references

\begin{tabular}{|c|c|c|c|c|c|c|c|c|}
\hline & \multicolumn{3}{|l|}{ Red } & \multicolumn{3}{|l|}{ Yellow } & \multicolumn{2}{|l|}{ Green } \\
\hline & R1P:R1 & R1P:R2 & R1P:R3 & Y1P:Y1 & Y1P:Y2 & Y1P:Y3 & G2P:G1 & G2P:G2 \\
\hline \multirow[t]{3}{*}{ GFC } & 0.9787 & 0.9367 & 0.9425 & 0.9866 & 0.9901 & 0.9684 & 0.9321 & 0.9953 \\
\hline & Orange 1 & & & Orange 2 & & & & \\
\hline & 01P:01 & 01P:02 & 01P:03 & O2P:O1 & O2P:02 & O2P:O3 & & \\
\hline GFC & 0.9407 & 0.9870 & 0.9671 & 0.9797 & 0.9680 & 0.9943 & & \\
\hline
\end{tabular}

The results in italics identify the highest value of GFC for each hue 


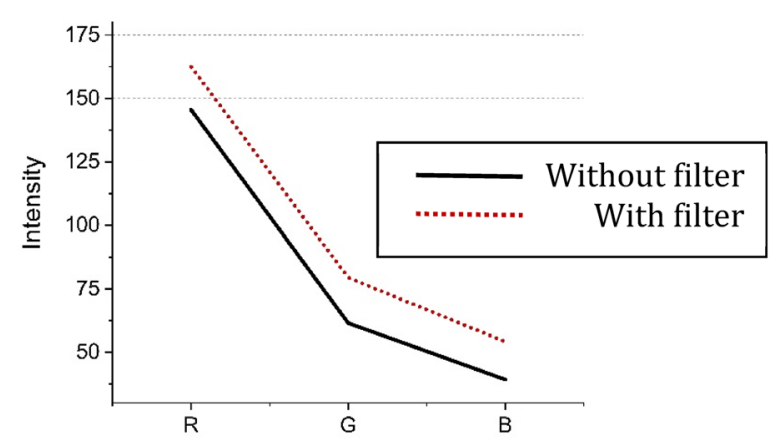

Fig. 10 Color channel of R1P with (dotted) and without (line) filter

pigment and the peak intensity. In other words, the pigment with less concentration and color strength would gradually fade. In the next step, the idea of manual filtering, which is a new method, was developed by fabricating filters of pigments. Identification was performed by imaging and calculating color channels, and then comparing their gradients with and without the filters. For pure pigments, when the filter was the same type as the pigment, the slope was identical. On the other hand, the slope changes with the variety in sample types. Finally, to evaluate the effectiveness of these methods for artworks, a sample painting was used. Then, by applying all the mentioned techniques and achieving the graphical and numerical results, its application as a non-destructive and profitable method was confirmed.

\section{Acknowledgements}

Not applicable.

\section{Authors' contributions}

FF collected database and analyzed samples. She interpreted data and was a major contributor in writing the manuscript. The whole paper was accomplished under Saeideh Gorji Kandi supervision. All authors read and approved the final manuscript.

\section{Funding}

The project did not receive funding.

\section{Availability of data and materials}

The datasets used and analyzed during the current study are available from the corresponding author on reasonable request.

\section{Competing interests}

The authors declare that they have no competing interest.

Received: 19 May 2020 Accepted: 13 September 2020

Published online: 24 September 2020

\section{References}

1. Casadio F, Toniolo L. The analysis of polychrome works of art: 40 years of infrared spectroscopic investigations. J Cult Herit. 2001;2(1):71-8.
2. Leona M, Casadio F, Bacci M, Picollo M. Identification of the pre-columbian pigment mayablue on works of art by noninvasive UV-Vis and raman spectroscopic techniques. J Am Inst Conserv. 2004;43(1):39-54.

3. J. K. Delaney, P. Ricciardi,L. D. Glinsman,M. Facini,M. Thoury,M. Palmer \&E. R. Rie, "Use of imaging spectroscopy, fiber optic reflectance spectroscopy, and X-ray fluorescence to map and identify pigments in illuminated manuscripts," vol. 59, no. 2, pp. 91-101, 2014

4. Cheilakou E, Troullinos M, Koui M. Identification of pigments on Byzantine wall paintings from Crete (14th century AD) using non-invasive Fiber Optics Diffuse Reflectance Spectroscopy (FORS). J Archaeol Sci. 2014;41:541-55.

5. Bonizzoni L, Bruni S, Gargano M, Guglielmi V, Zaffino C, Pezzotta A, Pilato A, Auricchio T, Delvaux L, Ludwig N. Use of integrated non-invasive analyses for pigment characterization and indirect dating of old restorations on one Egyptian coffin of the XXI dynasty. Microchem J. 2018;138:122-31.

6. Tortora M, Sfarra S, Chiarini M, Daniele V, Taglieri G, Cerichelli G. Nondestructive and micro-invasive testing techniques for characterizing materials, structures and restoration problems in mural paintings. Appl Surf Sci. 2016;387:971-85.

7. P. Colomban,"On-site Raman study of artwork: Procedure and illustrative examples," no. November, pp. 1-14, 2017.

8. Sfarra S, Castanedo C, Tortora M, Arrizzac L, Cerichelli G, Nardi I, Maldague X. Diagnostics of wall paintings: a smart and reliable approach. J Cult Herit. 2016;18:229-41.

9. S. Amookht, S. G. Kandi, M. Mahdavian, "Progress in Organic Coatings Mathematical description of spectrophotometric properties of metallic coatings using spectral derivation and principal component analysis," Prog. Org. Coatings, vol. 129, no. October 2018, pp. 338-348, 2019.

10. A. V. Agberien and B. Örmeci, "Monitoring of Cyanobacteria in water using spectrophotometry and first derivative of absorbance," 2020.

11. Cosentino A. Effects of different binders on technical photography and infrared reflectography of 54 historical pigments. Int J Conserv Sci. 2015;6(3):287-98.

12. Bacci M, Casini A, Cucci C, Picollo M, Radicati B, Vervat M. Non-invasive spectroscopic measurements on the II ritratto della figliastra by Giovanni Fattori: identification of pigments and colourimetric analysis. J Cult Herit. 2003;4(4):329-36.

13. Cavaleri T, Giovagnoli A, Nervo M. Pigments and mixtures identification by visible reflectance spectroscopy. Procedia Chem. 2013;8:45-54.

14. Dupuis G, Menu M. Quantitative evaluation of pigment particles in organic layers by fibre-optics diffuse-reflectance spectroscopy. Appl Phys A Mater Sci Process. 2005;80(4):667-73.

15. Leona M, Winter J. Fiber optics reflectance spectroscopy: a unique tool for the investigation of Japanese paintings. Stud Conserv. 2001;46(3):153-62.

16. M. Reháková, L. Gál, M. B. cová, M. Oravec, V. Dvonka, D. S. Cová, M. Ceppan, "Identification of iron-gall inks in historical drawings by Fibre Optics Reflection Spectroscopy-Extension to the NIR spectral range," J. Cult. Herit. vol. 27, pp. 137-142, 2017.

17. Wang J, Wu X, Xu Z. Potential-based obstacle avoidance in formation control. J Control Theory Appl. 2008;6(3):311-6.

18. Pan N, Hou M, Lv S, Hu Y, Zhao X, Ma Q, Li S, Shaker A. Extracting faded mural patterns based on the combination of spatial-spectral feature of hyperspectral image. J Cult Herit. 2017:27:80-7.

19. R. S. Berns and F. H. Imai, "The use of multi-channel visible spectrum imaging for pigment identification," 13th Trienn. Meet. Rio Janiero, 22-27 Sept. 2002 Prepr., pp. 217-222, 2002.

20. J. M. Fernández Rodríguez and J. A. Fernández Fernández,"Application of the second derivative of the Kubelka-Munk function to the semiquantitative analysis of Roman paintings," Color Res. Appl., vol. 30, no. 6, pp. 448-456, 2005

21. Barron V, Torrent J. Use of the Kubelka-Munk theory to study the influence of iron oxides on soil colour. J Soil Sci. 1986;37(4):499-510.

22. Szalai Z, Kiss K, Jakab G, Sipos P, Belucz B, Németh T. The use of UV-VIS-NIR reflectance spectroscopy to identify iron minerals. Astron Nachrichten. 2013;334(9):940-3.

23. Berns RS, Mohammadi M. Single-constant simplification of KubelkaMunk turbid-media theory for paint systems-A review. Color Res Appl. 2007;32(3):201-7.

24. Pallipurath AR, Skelton JM, Ricciardi P, Elliott SR. Estimation of semiconductor-like pigment concentrations in paint mixtures and their 
differentiation from paint layers using first-derivative reflectance spectra. Talanta. 2016;154:63-72.

25. Daniel F, Mounier A, Arantegui JP, Pardos C, Taboada NP, Vallejuelo SF, Castro K. Hyperspectral imaging applied to the analysis of Goya paintings in the Museum of Zaragoza (Spain). Microchem J. 2016;126:113-20.

26. Balas C, Epitropou G, Tsapras A, Hadjinicolaou N. Hyperspectral imaging and spectral classification for pigment identification and mapping in paintings by El Greco and his workshop. Multimed Tools Appl. 2018;77(8):9737-51

27. M. Alfeld, M. Mulliez, J. Devogelaere, L. de Viguerie, P. Jockey, and P. Walter, "MA-XRF and hyperspectral reflectance imaging for visualizing traces of antique polychromy on the Frieze of the Siphnian Treasury," Microchem. J., vol. 141, no. September 2017, pp. 395-403, 2018.

28. Grabowski B, Masarczyk W, Głomb P, Mendys A. Automatic pigment identification from hyperspectral data. J Cult Herit. 2018;31:1-12.

29. C. Barata, J. S. Marques, and J. Rozeira, "A System for the Detection of Pig ment Network in Dermoscopy Images Using Directional Filters," vol. 59, no. 10, pp. 2744-2754, 2012.
30. S. Baronti, A. Casini, F. Lotti, and S. Porcinai, "Multispectral imaging system for the mapping of pigments in works of art by use of principal-component analysis," vol. 37, no. 8, pp. 1299-1309, 1998.

31. R. Mazzeo, C. E. Palazzi, M. Roccetti, G. Sciutto, U. Bologna, and M. A. Zamboni, "Computer Assisted Pigment Identification in Artworks. Multispectral Scanner Imaging System " no. 1, pp. 1-6.

32. A. Cosentino, "Multispectral imaging of pigments with a digital camera and 12 interferential filters," e-Preservation Sci., vol. 12, pp. 1-7, 2015.

33. S. G. Kandi, "Representing Spectral Data Using Lab PQR Color Space in Comparison with PCA Method," vol. 4, pp. 95-106, 2011.

34. A. Cosentino, "FORS Spectral Database of Historical Pigments in Different Binders," e-conservation J., no. September, pp. 54-65, 2014.

\section{Publisher's Note}

Springer Nature remains neutral with regard to jurisdictional claims in published maps and institutional affiliations.

\section{Submit your manuscript to a SpringerOpen ${ }^{\circ}$ journal and benefit from:}

- Convenient online submission

- Rigorous peer review

- Open access: articles freely available online

- High visibility within the field

- Retaining the copyright to your article

Submit your next manuscript at $\boldsymbol{\Delta}$ springeropen.com 